\title{
Physical Layer-Aware Routing, Spectrum, and Core Allocation in Spectrally-Spatially Flexible Optical Networks with Multicore Fibers
}

\author{
Giannis Savva ${ }^{1}$, Georgios Ellinas ${ }^{1}$, Behnam Shariati $^{2}$, Ioannis Tomkos ${ }^{3}$ \\ ${ }^{1}$ KIOS CoE and Department of Electrical and Computer Eng., University of Cyprus, 1678 Nicosia, Cyprus \\ ${ }^{2}$ Universitat Politécnica de Catalunya (UPC), 08034 Barcelona, Spain \\ ${ }^{3}$ Athens Information Technology, Marousi, 15125 Athens, Greece \\ gsavva07@ucy.ac.cy
}

\begin{abstract}
In this work, efficient routing, spectrum, and core allocation (RSCA) techniques are proposed for spatial division multiplexed (SDM) elastic optical networks (EONs) utilizing multicore fibers (MCFs). These techniques provide efficient resource utilization with minimal computational complexity, while also taking into consideration physical layer effects. Initially, a crosstalk-aware approach is presented that accounts for the crosstalk effects in the network that can have detrimental impact on the connections established in the network. This approach is subsequently enhanced with a feedback-based procedure that takes into account all physical layer impairments, aiming to minimize the number of connections that cannot be established in the network due to quality-of-transmission (QoT) considerations.
\end{abstract}

\section{INTRODUCTION}

The exponential growth of high data rate traffic demands in backbone networks is expected to exceed the available capacity of traditional wavelength division multiplexed (WDM) networks. Flexible-grid networks, often called elastic optical networks (EONs), utilizing orthogonal frequency division multiplexing (OFDM), have been recently proposed by the research community to address this bandwidth crunch. In contrast to the fixed grid utilized in WDM networks, flexible-grid networks can now handle traffic demands by the elastic allocation of spectrum. For example, due to orthogonality, flexible-grid networks can now split the C-band into finer slices of 25, 12.5, and $6.25 \mathrm{GHz}$ compared to the $50 \mathrm{GHz}$ spacing of fixed grid networks. Thus, each demand is now allocated to a number of spectrum slices, called frequency slots, leading to a more efficient utilization of spectrum resources [1].

In traditional WDM networks, in order to assign a demand for a source-destination pair, first a path must be found, followed by a wavelength allocation along this path. This problem is the well known Routing and Wavelength Assignment (RWA) problem. Equivalently, in EONs, the Routing and Spectrum Assignment (RSA) problem must be solved, that includes finding a path (Routing) and a required spectrum assignment (SA) for the given demand. In the RSA problem, a feasible solution must satisfy several constraints: (i) the spectrum continuity constraint, where a demand must be allocated the same frequency slots on each link of the selected path, (ii) the non-overlapping constraint, which ensures that a frequency slot is allocated to one demand at a time, and (iii) the spectrum contiguity constraint, which implies that the number of frequency slots used to serve a demand have to be contiguous [2].

Even though elastic optical networks are expected to solve the capacity problem for the near term, due to the ever increasing development of new high data rate applications and services, it is anticipated that even these networks will at some point reach their limits in terms of transmission capacity. Thus, new technologies will be required in order to address future traffic demands [3]. Space Division Multiplexing (SDM) has been proposed as the ultimate solution to address the capacity crunch while reducing the cost-per-bit delivered to the endusers. However, in a long term horizon, it is anticipated that not only will standalone EON or SDM technologies revolutionize the Internet backbone, but also their combination: SpectrallySpatially Flexible Optical Networks (SS-FONs). SDMs can be implemented using a variety of optical fiber schemes such as multimode fibers (MMFs), few mode fibers (FMFs), multiple core fibers (MCFs), or bundles of single core fibers [2], [3]. In this work the focus will be on SS-FONs utilizing MCFs.

In SS-FONs, there are generally three mechanisms for switching from one core to another, which are highly correlated with the different types of fibers introduced for SDM networks [4]; independent switching (Ind-Sw), where all cores can be independently directed to any other core, joint switching $(J-S w)$, where each core can switch to only one core, and fractional joint switching ( $F r J-S w)$, a hybrid approach where a group of cores can be directed to another specific group of cores. Each switching mechanism offers benefits and drawbacks regarding performance, flexibility, and hardware cost as described in detail in [5].

It is an undeniable fact that SDM technology integration in optical communications offers many benefits through the expansion of spectral resources. However, physical-layer impairments must also be considered when a core assignment is made for a specific connection [6]. Crosstalk effects must especially be taken into consideration as they can potential have detrimental impact on a number of the connections 
established in the network. For the case of MCFs, inter-core crosstalk is the interference between adjacent cores, as during the signal transmission, a percentage of the signal travels into the cladding area creating evanescent waves and these waves can interfere with signals at the same frequency travelling in nearby cores. Assuming that an evanescent wave has low optical power, due to the small percentage leakage of the reflection as well as the exponential degradation due to the cladding medium, this interference is significant only between adjacent cores. The exact impact of crosstalk depends on the optical fiber's physical characteristics (e.g., number of adjacent cores and the distance between them), the number of allocated frequency slots operating at the same frequency in the adjacent cores, as well as the modulation format used at each frequency slot.

In SS-FONs utilizing MCFs, viable paths, available cores, and spectrum resources must be allocated for the establishment of the requested traffic demands (i.e., the routing, spectrum, and core allocation (RSCA) problem must be solved for each individual demand). In this case, all constraints of the RSA problem must also be followed, with the additional constraint that a connection that uses core switching is feasible as long as the same spectrum is occupied at the selected cores.

In this work, we focus on MCFs for a network planning scenario and we present a scalable heuristic algorithm to solve the routing, spectrum, and core allocation (RSCA) problem in SSFONs while also taking into account quality-of-transmission (QoT) constraints. We initially present a crosstalk-aware approach that aims to maximize efficiency in terms of resource allocation while minimizing the computation complexity during the allocation process. This approach is subsequently enhanced with a feedback-based procedure (utilizing a QoT estimator tool) that aims to minimize the number of connections that cannot be established in the network due to QoT considerations.

The rest of the paper is organized as follows. Section II provides a brief summary of the related work and Section III presents a physical layer-aware heuristic approach for solving the RSCA problem. Section IV presents the performance evaluation results of the proposed techniques, while Section V offers some concluding remarks.

\section{RELATED WORK}

As described in Section I, an efficient solution to the RSCA problem is critical in SS-FONs. Since the RSCA problem is NP-complete, authors in [7], [8] consider a planning scenario and formulate an ILP to optimally solve the RSCA problem for SS-FONs operating with MCFs. Specifically, in [7] the authors' objective is to increase the spectral efficiency of such networks, while in [8], the authors' objective is to minimize the maximum index of the spectrum slices allocated for all provisioned demands. Moreover, both approaches present heuristics to solve the RSCA problem by decomposing it into two sub-problems, namely the Routing (R) and the Spectrum and Core Allocation (SCA) subproblems. In [7], subcarriers in all connections are sorted in descending order based on their transmission distance and then spectrum and core allocation are performed for each connection while crosstalk interference is measured. However, in this work, the complexity of calculating crosstalk interference for a path can increase exponentially, considering that for a selected core in a path, all adjacent cores must be checked to measure the crosstalk interference. Further, in [8], the demands are sorted according to their spectrum requirements. Then, for each demand, all candidate paths are checked to determine the crosstalk interference and if lower than a given threshold, resources are allocated to the demand using the appropriate path.

To minimize computational complexity, authors in [9], [10] present various solutions having as objectives the efficiency and flexibility of the approaches for large sets of requests, while also considering crosstalk interference in a dynamic scenario. Specifically, in [9], the authors categorize cores based on the number of nearby cores in order to reduce crosstalk interference as well as spectrum fragmentation. They subsequently divide cores into groups, where each group is assigned to a specific demand size (e.g., 3-slot demands, 4-slot demands, etc.). Moreover, authors in [10] propose to "fuse" the spectrum and spatial dimension into virtualized resources to minimize computational complexity in the allocation process. Finally, in [11], the available spectrum, where crosstalk is above a given threshold, is found, and a virtual optical network embedding algorithm is proposed to solve the RSCA problem.

The reader should note that in all of the aforementioned techniques only crosstalk interference is considered, while any attempts made to consider the computational complexity of the proposed approaches fall short for realistic networks operating with multicore fibers. In the following section, a heuristic approach for the RSCA problem is presented, that efficiently allocates resources, while at the same time minimizing computational complexity as well as the number of connections that cannot be established in the network due to QoT considerations (including, in addition to crosstalk, other linear and nonlinear physical layer effects).

\section{PhysicAl LAYER-AWARE HEURISTIC APPROACH TO THE RSCA PROBLEM}

The proposed RSCA heuristic algorithm is divided into 2 subproblems: (i) the Routing subproblem, where $k$-candidate paths are found to serve a traffic demand and (ii) the Spectrum and Core Allocation subproblem, where resources are allocated to a given demand. Finally, a feedback-based approach is implemented to minimize the number of blocked connections that arise due to physical layer constraints.

\section{A. Routing}

In order to solve the Routing problem, a $k$-shortest path algorithm is developed that finds the "best" $k$ paths that can satisfy a given demand. Thus, more candidate paths are able to establish a connection for a given demand, leading to better overall results, since now more routes are checked before a requested connection is rejected. However, choosing the right criterion for sorting these $k$ candidate paths plays an important 
role in the performance of the system. One criterion would be the overall physical distance of a link in a given path, which results in the choice of the modulation format to be used for the entire path. Considering the constraints in the RSCA problem, each link in the chosen path must allocate the same number of frequency slots regardless of its distance in order to satisfy the continuity constraint, which means that the overall physical distance must be found and the appropriate modulation must be chosen based on that distance and applied for all the links in the path. Another criterion would be, for example, the number of hops in a path.

In this work, a hybrid method is utilized for sorting the group of $k$ paths, where both the modulation format and the number of hops are taken into account. Specifically, for the evaluation of each path, the ratio between the number of hops and the modulation ratio (number of bits mapped to a symbol) is considered. The motivation for the hybrid method is that both the modulation format and the number of hops are critical in deciding which routing path to use. Sorting the $k$ candidate paths in an ascending order based on this metric provides better results in terms of efficient spectrum utilization compared to the cases where either the number of hops or the available modulation format are used for sorting these paths. Further, since the proposed algorithm uses a preprocessing step to obtain and sort all available paths, compared to existing algorithms that calculate the spectrum needed using each path to serve a demand and then sorting the demands, the proposed technique outperforms existing techniques in terms of computational complexity.

In the following example, three different paths are shown for a requested connection from node 1 to node 5 . For simplicity, assume that, due to the modulation format used, the demand would require 9 frequency slots per link when using BPSK and 3 frequency slots per link when using 8-QAM. As shown in Table I, path $B$ is the best path if the number of hops is considered as the sorting criterion, whereas path $A$ would be the best solution if the overall path's length is the criterion for sorting the $k$ paths. However, as shown, the optimal solution would actually be path $C$ (utilizing the hybrid criterion) as it establishes a connection for the demand using the least spectrum resources (paths $A$ and $B$ would require 9 frequency slots, while path $C$ would only require 6 frequency slots (overall number of frequency slots $=$ number of frequency slots/link * number of links)).

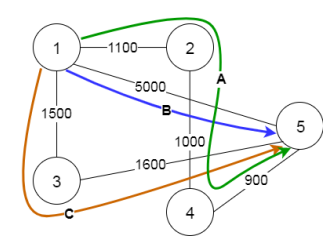

Fig. 1. Example of three different routing paths.
TABLE I

SORTING CRITERIA.

\begin{tabular}{|c|c|c|c|c|}
\hline Path & $\begin{array}{c}\text { Number of } \\
\text { Hops }\end{array}$ & $\begin{array}{c}\text { Modulation } \\
\text { Format }\end{array}$ & $\begin{array}{c}\text { Overall } \\
\text { Distance }\end{array}$ & $\begin{array}{c}\text { Hybrid } \\
\text { metric }\end{array}$ \\
\hline A & 3 & 8-QAM & 3000 & 1 \\
\hline B & 1 & BPSK & 5000 & 1 \\
\hline C & 2 & 8-QAM & 3100 & $2 / 3$ \\
\hline
\end{tabular}

\section{B. Spectrum and Core Allocation}

For the spectrum and core allocation (SCA) problem, a core classification step initially takes place in order to minimize crosstalk interference. In this step, a strict model is assumed where crosstalk occurs only when adjacent cores operate at the same frequency. Therefore, the spectrum is divided into groups and each core is allocated to different bandwidth resources from its adjacent ones. Thus, only the selected core's bandwidth must be checked before serving a given demand, also minimizing the computational complexity of the proposed method. Since crosstalk can partially exist in every connection (under a specified threshold), this procedure can be used until the utilized slots are saturated. Then, an algorithm can be utilized that checks the selected core and its adjacent ones to measure crosstalk before establishing a connection to serve any remaining demands.

To classify cores in an optimal way, crosstalk interference in a multicore fiber was modeled based on the distance between cores by generating an auxiliary graph where nodes and links represent the cores and the crosstalk effect between them, respectively (Fig. 2). Vertex coloring was subsequently used on the auxiliary graph to find the minimum number of different colors required to color all nodes without having same colored nodes next to each other (i.e., to obtain the core assignment while having no crosstalk between adjacent cores).

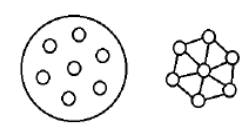

Fig. 2. The auxilary graph of a 7-core optical fiber.

The proposed algorithm uses a $N \times M$ matrix as input, namely the availability matrix, where $N$ is the number of cores and $M$ is the maximum number of wavelengths required. At a row $\mathrm{i}$ and column $\mathrm{j}$, " 1 " implies that wavelength $\mathrm{i}$ is used in core $\mathrm{j}$ and " $\mathrm{x}$ " implies that the specific wavelength cannot be used in the corresponding core. The number of nonempty rows signifies the number of wavelength groups used. Further, the geometry of the optical fiber is assumed to be known, which provides the distance between cores. Then, the categorization of each core is done as follows: (i) Step 1: a core is initially chosen and the first color is assigned to it, (ii) Step 2: available color matrix is updated so that the color assigned to that core will not be applied to its nearby cores, and (iii) Step 3: next core is chosen and assigned the first color that can be used without causing any interference. This procedure is repeated until all cores are assigned a color. In Fig. 3, a 
graphical representation of the aforementioned algorithm is presented.

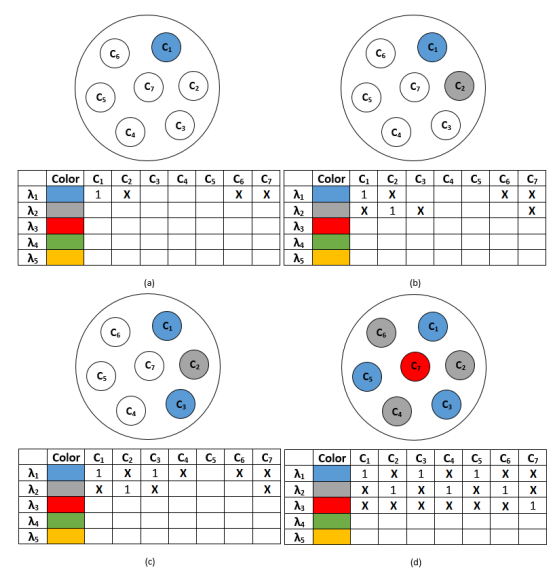

Fig. 3. (a) First core is chosen, wavelength $\lambda_{1}$ is assigned to it and availability matrix is updated. (b) Wavelength $\lambda_{2}$ is chosen for core 2 and the availability matrix is updated. (c) Core 3 is assigned to the first available wavelength, $\lambda_{1}$. (d) Core classification results after the termination of the algorithm.

Subsequently, to divide and assign bandwidth for each color, two approaches are followed: (i) the equal range division (ERD) technique, where the spectrum is divided into equal groups of frequency slots based on the number of colors obtained by the vertex coloring procedure, and (ii) the variable range division (VRD) technique, where spectrum is divided into variable groups of frequency slots based on the number of colors obtained from vertex coloring and the number of times each color appears. Fig. 4 graphically presents the results of these two approaches, for an example of a 7-core fiber, operating in the C-band, with spectrum slots of $25 \mathrm{GHz}$ spacing.
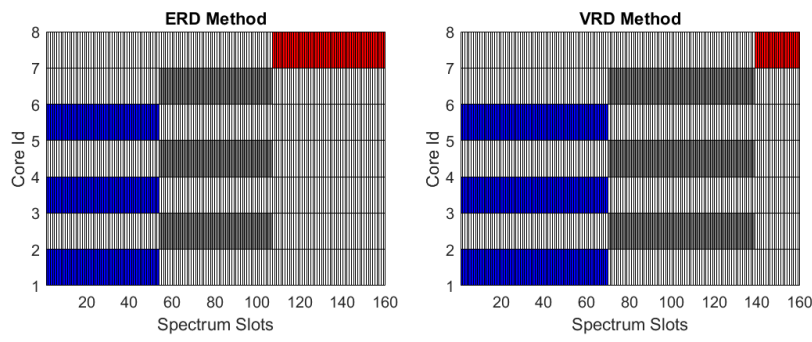

Fig. 4. 7-core fiber, $25 \mathrm{GHz}$ channels - ERD and VRD methods.

In the following table, the number of utilized frequency slots are presented for several multi-core fibers that can be used in SS-FONs.

TABLE II

FREQUENCY SLOTS REQUIRED FOR ERD AND VRD METHODS.

\begin{tabular}{|c|c|c|}
\hline $\begin{array}{c}\text { Type of } \\
\text { Multicore Fiber }\end{array}$ & $\begin{array}{c}\text { Number of freq. slots: } \\
\text { ERD Method }\end{array}$ & $\begin{array}{c}\text { Number of freq. slots: } \\
\text { VRD Method }\end{array}$ \\
\hline 7-core & 372 & 436 \\
\hline 10-core & 400 & 544 \\
\hline 19-core & 760 & 848 \\
\hline
\end{tabular}

It is important to note that the ERD method can be used to efficiently reduce fragmentation if each equally divided group of frequency slots serves specific connection sizes (e.g., blue for 3-, gray for 4- and red for 5-slot demands respectively as shown in Fig. 4 above). Nevertheless, Fig. 4 and Table II demonstrate that using the VRD method provides better results in terms of available frequency slots to accommodate all requested connections without any crosstalk interference. This is the case since this technique also takes into account the number of times each color appears, thus dividing the spectrum more efficiently. Further, it is also important to note that both methods significantly reduce computational complexity, since now the core operates at different frequency slots compared to its adjacent ones, thus, checking bandwidth in adjacent cores is no longer necessary.

\section{Feedback-based Process: QoT-aware Approach}

In the previous section we described an SCA approach that takes only inter-core crosstalk into consideration. In order to account for additional physical layer impairments that are present in the network as well (both linear and nonlinear effects), the Quality of Transmission (QoT) estimator tool presented in [6] is used to evaluate all paths allocated utilizing the aforementioned algorithm. The QoT estimator module predicts the QoT of new lightpaths to be established in the network as well as the impact on existing connections when setting up a new one, by taking into account the amplified spontaneous emission (ASE) noise and analytical models of linear and nonlinear impairments of SDM fibers. The tool input comprises of the network topology, spectral windows, link characteristics, signal types (baud rate and modulation format), as well as lightpaths currently established in the network. As, in practice, there are multiple established lightpaths resulting in network links accommodating a different number of channels from different end-to-end connections, for a given lightpath the signal related characteristics are not the same for each link and depend on the already established connections.

In order to account for the physical layer impairments, a feedback process is applied as follows: The QoT estimator tool is used to evaluate the connections established by the algorithm. If the BER of a connection is below a specific predefined BER threshold, it is considered as blocked. Then, all blocked demands are reallocated by the proposed algorithm (excluding the previously found path and repeating the RSCA approach described in Sections III-A and III-B above) and the QoT estimator tool re-estimates all paths (this is the case as new connections may affect previously established connections). Finally, the process terminates if the BER of all demands established by the proposed algorithm are above the BER threshold or the number of process repetitions is above a pre-defined threshold.

Note that due to the low computational complexity of the approach in solving the RSCA problem, the process of reallocating resources for the given demands still retains very low computational complexity. 


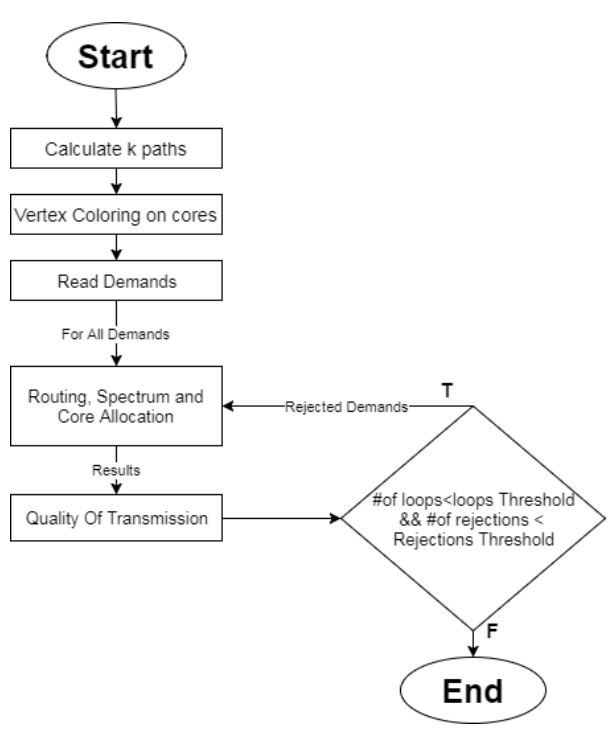

Fig. 5. Flowchart of the feedback process using the QoT estimator tool.

\section{Performance Evaluation}

The simulation setup used to evaluate the proposed algorithm is as follows: the elastic optical network is implemented using bandwidth variable transponders (BV-Ts) that operate using multiple modulation formats: BPSK, QPSK, 8-QAM, and 16-QAM. The transmission reach for each modulation format is given by $9300,4600,1700$, and $800 \mathrm{~km}$ respectively. Moreover, a flexible grid is implemented with channel spacing of $25 \mathrm{GHz}$ and baud rate of $16 \mathrm{GBaud}$. For the performance evaluation, two network topologies are used; the Telefonica network which consists of 30 nodes and 56 undirected links and the US network with 24 nodes and 43 undirected links. In all cases, demands are randomly generated using a uniform distribution (all source-destination pairs are equally likely and each demand size varies from 20 to 200Gbps). Each experiment is performed for 5 sets of demands and the average of these results is presented.

First, the results of the three different criteria utilized for sorting the candidate paths are presented. To evaluate the impact of choosing each sorting criteria, the feedback process is not applied. As shown in Fig. 6, the Hybrid method gives the best results in terms of spectrum utilization, using less spectrum slots to serve the same amount of demands. Using Max Length as a criterion results in the best modulation format usage; however, if the number of hops is large for a given path, the overall number of frequency slots required for that demand will increase. Thus, as demonstrated, the consideration of both parameters increases the efficiency of the system.

To minimize crosstalk interference between cores, each core has to operate at a different wavelength compared to its adjacent ones. The optimal crosstalk-aware solution would be to check the selected core and all of its adjacent ones for all frequency slots the demand needs, while considering the necessary constrains the RSCA imposes, as well as the specified crosstalk threshold. In this work, this approach is

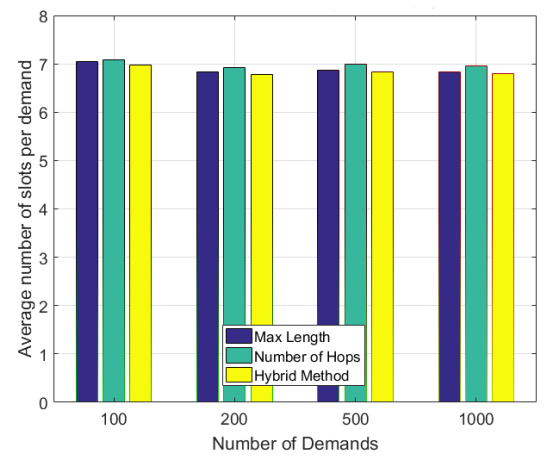

Fig. 6. Route sorting evaluation.

referred to as the Slot per Slot ( $\mathrm{SpS}$ ) algorithm. This is however a greedy approach, where computations can grow significantly based on the switching mechanism, the number of demands, the decision of how many shortest paths to consider, and the size of network.

As the computational complexity of the RSCA problem can grow significantly when solved utilizing the $\mathrm{SpS}$ approach, the proposed and $\mathrm{SpS}$ algorithms are compared in terms of execution times. Considering that the $\mathrm{SpS}$ algorithm does not minimize fragmentation, which is the main benefit for using the ERD method, the VRD method is chosen for all follow up experiments. Also, to measure the difference between using the SpS algorithm and the proposed algorithm, the feedback process execution times are not considered in these experiments.

To magnify the difference between the proposed and the SpS approach and demonstrate the scalability of the proposed technique, several experiments were performed for both topologies with 19-core optical fibers using independent switching and 7 candidate shortest paths (note that the $k$ shortest path algorithm execution time is not accounted for in the calculations so as to show the exact difference between the two approaches). All simulations were executed on a PC with an Intel Core $i 7-4500 U 2.4 \mathrm{GHz}$ CPU and 8GB RAM. In Fig. 7 that follows, computational times are presented for the SpS algorithm and the proposed algorithm using the VRD approach, clearly demonstrating the superiority of the proposed technique that exhibits low computational times even for large numbers of requests.

To evaluate the proposed (crosstalk-aware) approach without utilizing the feedback process, the QoT estimator tool is utilized. For the evaluation of the proposed algorithm, 7-core fibers were considered using the J-Sw switching mechanism and the Telefonica network topology. Further, channels with $25 \mathrm{GHz}$ spacing are utilized with a baud rate of 16 Gbaud. The BER threshold for rejecting a connection was set to $10^{-3}$ as also described in [6]. Figure 8 presents the results of the proposed algorithm with all three sorting criteria using the QoT estimator tool, illustrating the advantage of utilizing the proposed hybrid approach.

Finally, we evaluate the RSCA physical layer-aware ap- 


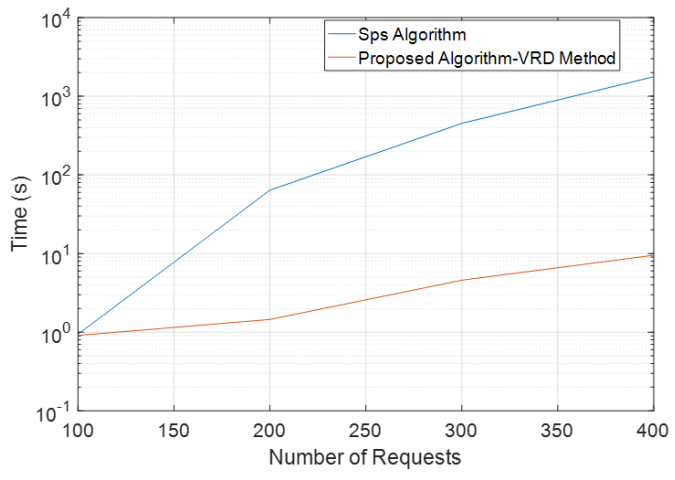

Fig. 7. Execution times - SpS vs VRD techniques.

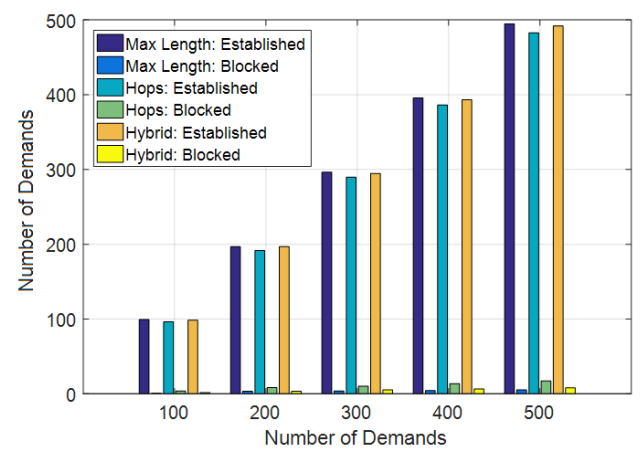

Fig. 8. Quality-of-transmission results: Crosstalk-aware algorithm.

proach, where the feedback-based process takes place utilizing the proposed RSCA algorithm and the QoT estimator tool to obtain the best results in terms of spectrum efficiency, computational complexity, and physical impairment awareness in SS-FONs. For this set of experiments, we considered the best results obtained from the aforementioned simulations. Thus, the proposed algorithm with the VRD method is implemented in an SS-FON using 7-core fibers with $25 \mathrm{GHz}$ spacing, 16 Gbaud frequency slots, and the J-sw switching mechanism. Further, the Hybrid method is used to sort the $k$-candidate paths. Figure 9 demonstrates the results of the feedback-based process for different feedback loop thresholds. As shown in the figure, using the feedback-based process significantly reduces the blocking probability for each set of demands (approximately 5 repetitions are shown to be enough to minimize as much as possible the blocking rate of the connections that cannot be established due to QoT considerations). Further, it is also important to note that after a certain number of demands, the feedback process is not advantageous in reducing the blocking probability, as now the limitation is the number of available resources rather than the algorithms used.

\section{CONCLUSiOnS}

This work presents a novel crosstalk-aware RSCA algorithm, utilizing a hybrid (distance and number of hops) method as a routing sorting criterion and the variable range division

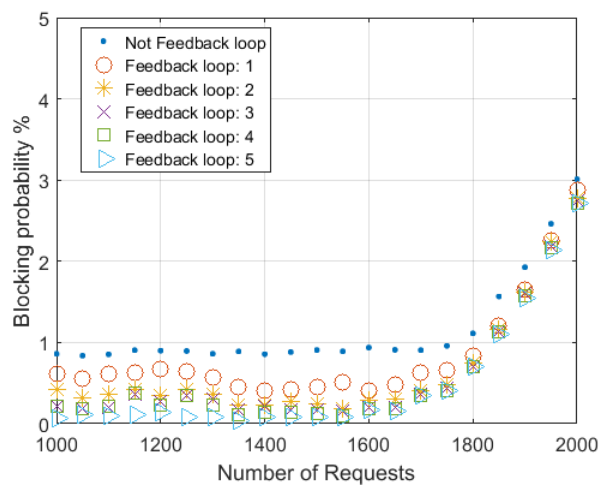

Fig. 9. Blocking probability versus number of requests: Physical layer-aware approach.

(VRD) core classification method that was shown to outperform the traditional SpS approach in terms of spectrum efficiency, as well as in terms of computation complexity. This technique was further validated by a QoT estimator tool demonstrating the feasibility of the connections established. A feedback-based process was also demonstrated that allowed the minimization of the blocked connections due to physical layer impairment considerations, via the utilization of the proposed RSCA technique in conjunction with the QoT estimator tool.

\section{ACKNOWLEDGMENT}

This work has been supported by the European Unions Horizon 2020 research and innovation programme under grant agreement No 739551 (KIOS CoE) and from the Government of the Republic of Cyprus through the Directorate General for European Programmes, Coordination and Development.

\section{REFERENCES}

[1] O. Gerstel, et al., "Elastic Optical Networking: A New Dawn for the Optical Layer?”, IEEE Comm. Magazine, 50(2):S12-S20, 2012.

[2] A. Muhammad, et al., "Resource Allocation for Space Division Multiplexing: Optical White Box vs. Optical Black Box Networking”, IEEE/OSA J. of Lightw. Technology, 33(23):4928-4941, 2015.

[3] D.J. Richardson et al., "Space-division Multiplexing in Optical Fibers", Nat. Photon., 7(5):354-362, 2013.

[4] B. Shariati et al, "Impact of Spatial and Spectral Granularity on the Performance of SDM Networks based on Spatial Superchannel Switching," IEEE/OSA J. of Lightw. Technology, 35(1):2559-2568, Jul 2017.

[5] B. Shariati et al, "Evaluation of the Impact of Different SDM Switching Strategies in a Network Planning Scenario", Proc. OFC, 2016.

[6] B. Shariati et al., "Spectrally-Spatially Flexible Optical Networking", Proc. ACP, 2016.

[7] M. N. Dharmaweera, et al., "Nonlinear-Impairments- and CrosstalkAware Resource Allocation Schemes for Multicore-Fiber-based Flexgrid Networks", Proc. ECOC, Dusseldorf, Germany, 2016.

[8] A. Muhammad et al, "Routing, Spectrum and Core Allocation in Flexgrid SDM Networks with Multi-core Fibers", Proc. ONDM, 2014.

[9] S. Fujii, Y. Hirota, H. Tode and K. Murakami, "On-Demand Spectrum and Core Allocation for Reducing Crosstalk in Multicore Fibers in Elastic Optical Networks", IEEE/OSA J. Optical Communications and Networking, 6(12):1059-1071, 2014.

[10] H. Tode and Y. Hirota, "Routing, Spectrum, and Core and/or Mode Assignment on Space-Division Multiplexing Optical Networks", IEEE/OSA J. Optical Communications and Networking, 9(1):A99-A113, 2017.

[11] R. Zhu, et al., "Crosstalk-aware Virtual Optical Network Embedding (VONE) in Spatial Division Multiplexing Enabled Elastic Optical Networks with Multi-core Fibers", Proc. ECOC, Dusseldorf, Germany, 2016. 